\title{
Postinfarction left ventricular remodelling: where are the theories and trials leading us?
}

\author{
Z R Yousef, S R Redwood, M S Marber
}

Table 1 Components of postinfarction ventricular remodelling

- Infarct expansion - Neurohormonal activation - Myocardial hypertrophy

- Global ventricular dilatation

\author{
Department of \\ Cardiology, The Rayne \\ Institute, St Thomas' \\ Hospital, King's \\ College London, \\ London SE1 7EH, UK \\ Z R Yousef \\ M S Marber \\ S R Redwood \\ Correspondence to: \\ Professor Marber \\ email: mike.marber@ \\ kcl.ac.uk \\ Accepted for publication \\ 21 July 1999
}

While mortality from overt ischaemic heart disease is falling, that from heart failure is increasing and reaching epidemic proportions. ${ }^{1}$ Although an aging population is partly responsible for this trend, recent observations showing persistently high mortality and morbidity rates following myocardial infarction further compounds the issue. ${ }^{2}$ An understanding of the pathophysiological processes leading to heart failure, and in particular the mechanisms underlying postinfarction heart failure (postinfarction ventricular remodelling) is therefore fundamental and forms the basis of this review.

\section{Acute myocardial infarction}

Immediately after coronary artery occlusion, irreversible cell necrosis can occur within minutes. Factors affecting the amount of necrosis include the presence or absence of a preconditioning stimulus, ${ }^{3}$ the volume of ischaemic myocardium, and the amount of collateral blood flow. ${ }^{4}$ In addition, prompt reperfusion within a narrow time window, when myocytes are in a state of critical ischaemia and are prenecrotic/viable, has been shown to reduce cell death, limit infarct size, and increase survival. ${ }^{5}$

Myocardial infarction is followed by a complex and interrelated sequence of events termed postinfarction left ventricular remodelling (table 1) which describes the compensatory responses of the cardiovascular system when faced with an acute loss of myocardial contractile function. ${ }^{6}$ Morphologically, the end result of these responses can be quantified by imaging the infarcted ventricle as it progressively dilates and assumes a more spherical as opposed to elliptical contour. ${ }^{6}$

In the early stages, ventricular dilatation can be considered beneficial as it maintains stroke volume through the Frank-Starling mechanism. At the same time, however, the dilating ventricle proves detrimental as it exerts further demands on the surviving myocardium through increases in wall tension (the law of Laplace, fig 1). ${ }^{7}$ Long term, left ventricular volume is a sensitive marker of postinfarction ventricular dysfunction, left ventricular end systolic volume being one of the most powerful independent predictors of prognosis after myocardial infarction. ${ }^{8}$

\section{Infarct expansion}

Cell necrosis initiates an inflammatory reaction with granulocyte infiltration and release of proteolytic enzymes. The matrix metalloproteinases (MMP), a family of zinc dependent collagenases and their endogenous inhibitors (tissue inhibitors of MMP, TIMP) play a crucial role in subsequent collagen degradation and infarct expansion. ${ }^{9}$ Collagen breakdown predominates up to 14 days following infarction when serum levels of $\mathrm{MMP}-1$ are raised. Thereafter, serum TIMP-1 concentrations prevail and activity shifts from lysis to fibroblast infiltration, collagen deposition, and scar formation. ${ }^{10}$

During the early lytic phase, the infarcted segment is vulnerable to pressure and shape change within the ventricle which increase wall stress and strain and result in lengthening and thinning through lateral slippage of myocardial planes. ${ }^{11}$ The surface area occupied by the infarcted region thus increases and the infarct zone expands. The magnitude of infarct expansion depends on various factors (table 2), which, when extreme, can lead to left ventricular or septal rupture.

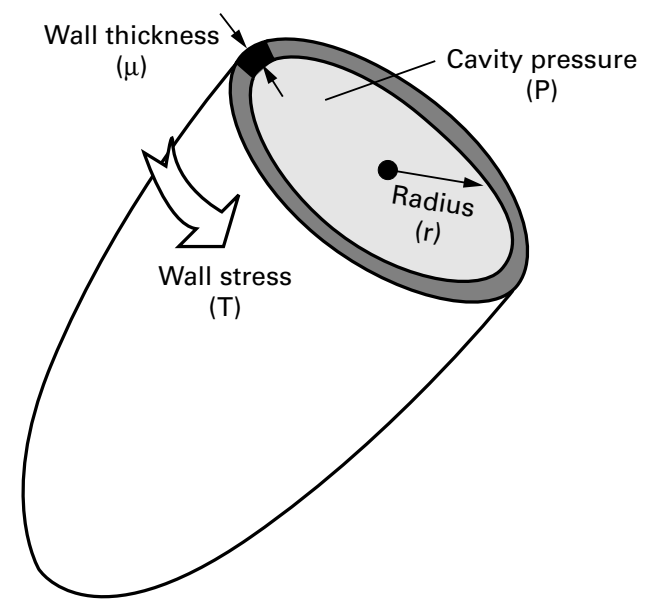

The law of Laplace:

Wall stress $(T)=[$ cavity pressure $(P)] \times[\operatorname{radius}(r)]$ $2 \times[$ wall thickness $(\mu)]$

Thus as left ventricular cavity pressure increases (eg, aortic stenosis, hypertension), the wall thickness increases (hypertrophy) to maintain wall stress. Furthermore, as the cavity dilates (infarct expansion), wall stress increases to maintain cavity pressure.

Figure 1 The law of Laplace. 
Table 2 Factors associated with infarct expansion

Aggravating factors
- Anterior site of infarction
- Raised intraventricular pressure
- Concurrent anti-inflammatory treatment (steroids,
NSAID)
Limiting factors
- Non-Q wave myocardial injury
- Pre-existing ventricular hypertrophy infarction)
NSAID, non-steroidal anti-inflammatory drugs.

\section{Neurohormonal activation}

Plasma concentrations of several neurohormones are increased after myocardial infarction. The magnitude and time course of activation are closely related to infarct size and degree of left ventricular dysfunction. ${ }^{12}$ The neurohormonal hypothesis postulates that neurohormones initially serve an adaptive role by maintaining cardiac output, but in the later stages the responses becomes pathological and contribute to adverse remodelling, progressive ventricular failure, and ultimately to the syndrome of heart failure. ${ }^{13}$

\section{AUTONOMIC SYSTEM}

One of the earliest responses during infarction is an increase in sympathetic drive which has both positive chronotropic and inotropic actions. While cardiac output is maintained, left ventricular loading increases, thereby aggravating infarct expansion and promoting adverse ventricular remodelling. In addition, myocardial oxygen demand rises, precipitating further ischaemia, and the proarrhythmic effects of catecholamines may predispose to fatal arrhythmias.

In the long term, sympathetic activation contributes to the re-expression of a fetal gene program and ventricular hypertrophy which may ultimately exacerbate ventricular dilatation. ${ }^{14}$ The corollary that sympathetic antagonists confer advantage has been shown in several randomised $\beta$ blocker trials including a meta-analysis which concluded that treatment with $\beta$ adrenoceptor blocking drugs for 12 months after myocardial infarction reduces mortality by up to $25 \% .^{15}$ More recently, vasodilating $\beta$ blockers have been shown to reduce the risk of all cause mortality in established heart failure by up to $65 \%$ after just six months of treatment, ${ }^{16}$ while in another similar randomised, double blind, placebo controlled study, the addition of a conventional $\beta$ blocker (bisoprolol) to "standard" medical treatment resulted in a comparable reduction in all cause mortality (34\%) following 18 months of treatment. ${ }^{17}$

THE RENIN-ANGIOTENSIN AXIS

Vasoconstriction and fluid retention are both actions of the renin-angiotensin axis which serve to maintain blood pressure. In common with catecholamines, left ventricular afterload eventually rises and adverse remodelling is promoted. A reduction in the activity of this axis with angiotensin converting enzyme (ACE) inhibitors thus appears appropriate and forms the rationale for the large ACE inhibitor trials. Such mechanisms are likely to underlie the $11 \%$ reduction in mortality after myocardial infarction observed at six weeks in the GISSI-3 trial (Gruppo Italiano per lo Studio della Sopravvivenza Nell'Infarto Miocardico), where ACE inhibitors were used in the first 24 hours of infarction. ${ }^{18}$ Similar processes may explain the late benefits of ACE inhibitors after infarction. For example, a $21 \%$ risk reduction of cardiovascular mortality was observed after an average follow up of 42 months in patients treated with captopril (started three to 16 days after myocardial infarction) compared with placebo matched controls. ${ }^{19}$ Comparable benefits of enalapril are also seen in patients with established left ventricular dysfunction (but not necessarily a history of myocardial infarction), with a $22 \%$ reduction in death from progressive heart failure after a mean follow up of 41 months. $^{20}$ These effects might reflect a reduction of systemic angiotensin II, which, in addition to its hypertensive effects, has various other detrimental myocardial actions. ${ }^{21}$

More recently, antagonists of the angiotensin II type 1 receptor (AT1-R) have been developed. The ELITE study (evaluation of losartan in the elderly) compared captopril (an ACE inhibitor) with losartan (an AT1-R antagonist) in elderly, ACE-I naive patients. ${ }^{22}$ Although the trial was designed to examine a primary end point of renal tolerability, a trend towards a reduction in all cause mortality in the losartan group was observed $(4.8 \%$ \% $8.7 \%$ after 48 weeks of treatment)..$^{22}$ ELITE II, an ongoing trial designed to examine all cause mortality, may further our understanding of the mechanisms underlying postinfarction ventricular remodelling including the relative actions played by the kinins, for example bradykinin.

\section{NATRIURETIC PEPTIDES}

The three principal members of the natriuretic peptide family (atrial natriuretic factor, ANF; brain natriuretic peptide, BNP; and C-terminal of atrial natriuretic peptide, CNP) exert their phenotypic effects through two main receptors (A and B). Systemic levels of the peptides are increased following myocardial infarction, with ANF being released from the right atrium in response to increases in right atrial stretch, and $\mathrm{BNP}$ (and to a lesser degree CNP) being released from the ventricles in response to raised right ventricular wall tension. ${ }^{23} \mathrm{ANF}$ and BNP exert opposite effects to the sympathetic outflow and the renin-angiotensin axis by reducing afterload through a combination of peripheral vasodilatation and natiuresis. ${ }^{23}$ In addition to their early beneficial effects on haemodynamics, fluid balance, and renal function, there is experimental evidence that the natriuretic peptides may in the long term inhibit myocyte hypertrophy and therefore foster an environment for beneficial remodelling. ${ }^{24}$

However, their use clinically has so far been unsuccessful. The peptides have a short half life, and tolerance to their effects develops rapidly. Furthermore, potent hypotension and bradycardia have limited their use, though novel approaches involving TIMP inhibiting natriuretic peptide clearance are underway in experimental models. In a recent study, for 

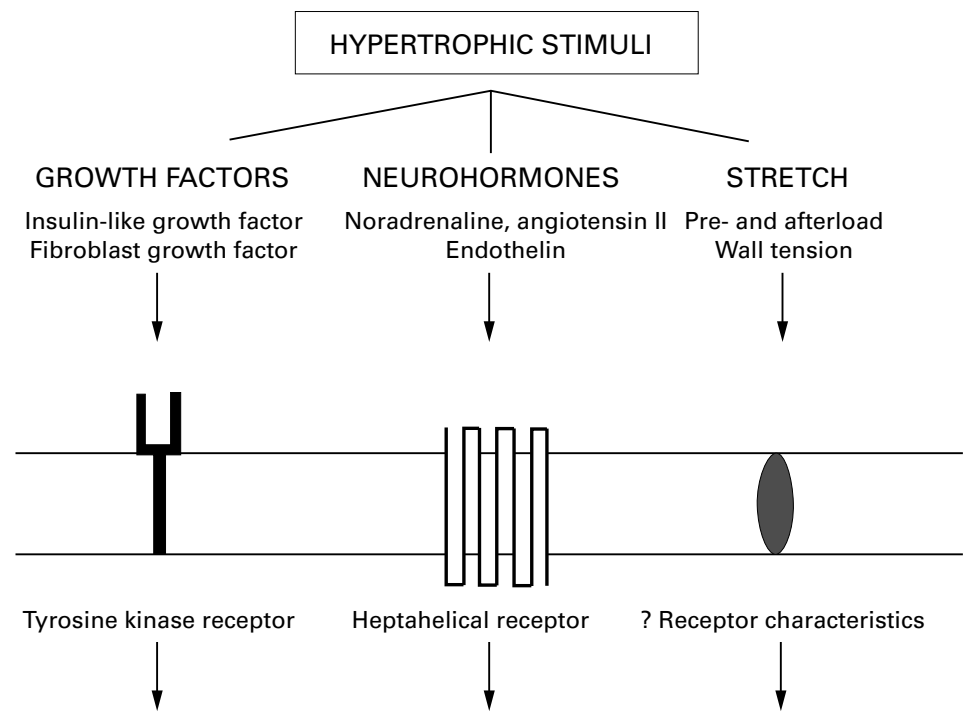

Ras/Raf pathway Phosphoinositol pathway Calcium associated pathway

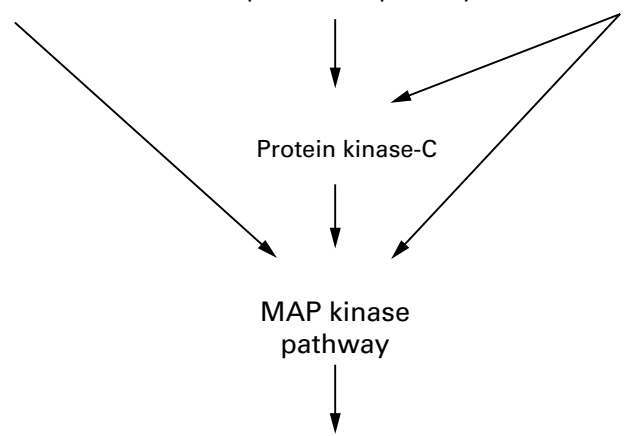

NUCLEAR TRANSCRIPTION

Figure 2 Signalling pathways associated with myocardial hypertrophy. A simplified overview of our current understanding of the intracellular signalling pathways leading to myocardial hypertrophy. Although the molecular events coupling membrane receptors to nuclear events have not been fully documented, three principal arms (with interpathway crosstalk) are described, each converging onto a common pathway (mitogen activated protein kinase cascade) which ultimately leads to the nucleus. Considerable work remains to be done to fully characterise the pathways before any potential therapeutic targets can be identified. studies. In a postinfarction rat model of heart failure, long term treatment with bosentan (a combined $\mathrm{A}$ and $\mathrm{B}$ receptor antagonist) resulted in benefical remodelling compared with placebo matched controls. ${ }^{28}$ In addition to a significant survival benefit in the bosentan group $(65 \%$ v $47 \%)$, active treatment also resulted in improved haemodynamic stability, a reduction in plasma catecholamines, and reduced left ventricular collagen density (myocardial fibrosis). ${ }^{28}$ Furthermore, ex vivo analysis after two months of treatment showed a reduction in left ventricular dilatation and hypertrophy. ${ }^{28}$

Human studies with bosentan, however, are limited through concerns over safety. For example, the REACH-1 trial (research on endothelin antagonism in chronic heart failure) was terminated prematurely when an unprecedented proportion of patients receiving bosentan developed raised plasma transaminases. ${ }^{29}$ Although only $47 \%$ of patients completed the study, bosentan treatment resulted in a significant clinical improvement, a reduction in symptomatic deterioration, and a reduction in the total number of hospital admissions by $40 \%{ }^{29}$

Thus although interruption of the endothelin pathway is an attractive target with potential advantages, long term pharmacological studies with non-toxic agents are needed before phase III trials can occur in patients with cardiac failure and hypertension.

\section{Myocardial hypertrophy}

Adult myocytes are highly specialised terminally differentiated cells and as such have probably lost the ability to divide. Their response to an increased workload is an increase in size and protein synthesis, in addition to an increased production and assembly of sarcoplasmic contractile units. ${ }^{30}$ The hypertrophic stimuli consist of neurohormonal agents (for example, noradrenaline, angiotensin II, endothelin), local growth promoting peptides (insulin-like growth factor I, cardiotrophin I, fibroblast growth factor), and physical factors causing myocyte stretch (increased preload and afterload, increased wall tension). ${ }^{14}$ The stimuli act on specific cell surface receptors which activate a cascade of intracellular signalling pathways (fig 2).

Minutes after membrane receptor activation, induction of early response genes (for example, c-fos, c-jun, and c-myc) may be observed. ${ }^{31}$ These genes (also termed proto-oncogenes) are functionally analogous to virus associated oncogenes and are responsible for the synthesis of small regulatory proteins which control the transcription of other genes. There follows a re-expression of a fetal gene program, which in animal models includes the induction of contractile proteins ( $\beta$ myosin heavy chain, $\alpha$ skeletal actin, and $\beta$ tropomyosin), and noncontractile proteins (ANF and $\beta 2 \mathrm{Na} / \mathrm{K}-$ ATPase) which ordinarily are only detectable in the fetal period when global cellular hyperplasia predominates. ${ }^{32} \mathrm{~A}$ similar reversion to the fetal genome may be observed in adult human cells capable of division when stimu- 
lated appropriately (for example, the reexpression of $\alpha$ fetoprotein by acutely damaged hepatocytes following infective hepatitis). ${ }^{33}$ It appears therefore that adult myocytes may experience similar molecular signals to other cells when faced with a noxious stimulus, but whereas other cells are able to respond by mitosis, myocytes which are arrested in the $G_{0}$ phase of the cell cycle undergo hypertrophy which appears to be the only growth response available.

\section{Global ventricular dilatation}

In addition to early left ventricular dilatation (infarct expansion), the ventricle undergoes a later and more insidious process of further dilatation when other compensatory mechanisms fail to maintain cardiac output. ${ }^{6}$ In general, where the infarcted region occupies over $10 \%$ of the total myocardial mass (as occurs in approximately $40 \%$ of all transmural anterior myocardial infarctions) the ventricle progressively enlarges, heralding the onset of symptomatic heart failure and its associated hazards. ${ }^{34}$

Initially, hypertrophy involves the serial deposition of new sarcoplasmic elements, which results in elongation of cells without an increase in cell thickness (eccentric hypertrophy). ${ }^{6}$ This pattern of hypertrophy causes global left ventricular dilatation and wall thinning which increases wall tension (the law of Laplace). ${ }^{7}$ In addition, these cardiocytes may have an impaired contractility which is further compounded by the greater left ventricular mass to cavity volume ratio, leading to relative ischaemia as the capillary network cannot keep pace with tissue growth and is unable to support the greater demands of the hypertrophied tissue. ${ }^{6}$

Ultimately a vicious cycle is established where loss of contractile tissue activates several adaptive mechanisms which, when unable to compensate adequately, lead to persistently abnormal loading conditions. These in turn exaggerate the compensatory responses which have already failed. The earlier beneficial effects now become maladaptive and the abnormal loading conditions are further enhanced, thus fuelling the downward spiral that perpetuates ventricular dysfunction. The end result is a progressive dilatation of the left ventricular leading to symptomatic heart failure and ultimately death.

\section{Intervention and postinfarction ventricular remodelling}

The advantages gained from reperfusing viable tissue are undisputed. ${ }^{5}$ However, the potential benefits of recanalising vessels supplying an infarcted and non-viable region remain controversial. ${ }^{35}$ In animal models, late reperfusion following experimental myocardial infarction at a point beyond myocardial salvage significantly reduces infarct expansion and benefits remodelling. ${ }^{36}$ In humans, observational studies in the postinfarction period have consistently shown survival benefits in the presence of a patent infarct related artery. ${ }^{37}$ Studies randomising patients after myocardial infarction to medical treatment with or without intervention are, however, few and conflicting. In the SWIFT study (Should We Intervene Following Thrombolysis?), patients surviving acute myocardial infarction were randomised to either conservative care or early angiography plus intervention for patients with stenotic but non-occlusive disease of the infarct related artery. ${ }^{38} \mathrm{~A}$ trend towards increased death or non-fatal reinfarction one year later was shown in the interventional group $(19.1 \% v 16.6 \%$; odds ratio 1.19$).{ }^{38}$ In a similar study with over 3000 patients, no differences were observed between patients managed aggressively or conservatively (combined rate of death and non-fatal myocardial infarction 12 months after myocardial infarction $14.7 \% v 15.2 \%$, respectively). ${ }^{39}$ However, a more recent study randomising 83 patients after anterior myocardial infarction to medical treatment with or without PTCA reported a significant reduction in left ventricular end systolic volume at six months, and increased five year cardiac eventfree survival in the PTCA group. ${ }^{40}$ Thus although interventional strategies aimed at either halting or reversing adverse postinfarction remodelling are attractive, data supporting these approaches remain speculative and we must await the results of ongoing multicentre studies investigating the "open artery hypothesis" $" 35$ in order to determine whether there is long term benefit in opening occluded coronary arteries beyond the time window of salvage.

\section{Conclusions}

The socioeconomic burden of heart failure is projected to increase. Current treatment, which includes ACE inhibitors and $\beta$ blockers, only partially reduces the high mortality, which is similar five years after presentation to that of many malignancies. An increase in prevalence of heart failure and an increase in diseased rather than disease free survival will combine to increase the burden of this condition on society.

Further interventions aimed at reducing infarct size, supporting the extracellular matrix, modulating neurohormones, and producing regression of maladaptive cellular hypertrophy are needed if we are to improve the long term prognosis and reduce the morbidity of patients after myocardial infarction. In addition, the role of intervention in this setting requires clarification through additional randomised clinical trials.

ZY, SRR, and MSM are supported in part by The British Heart Foundation.

1 Garg R, Packer M, Pitt B, et al. Heart failure in the 1990s: volution of a major public health problem in cardio cular medicine. F Am Coll Cardiol 1993;22(suppl A):3-5A.

2 Mahon NG, O'Roke C, Codd MB, et al. Hospital mortality of acute myocardial infarction in the thrombolytic era. Heart 1999;81:478-82.

3 Murry CE, Jennings RB, Reimer KA. Preconditioning with ischemia: a delay of lethal cell injury in ischemic myocardium. Circulation 1986;74:1124-36.

4 Habib GB, Heibig J, Forman SA, et al. Influence of coronary collateral vessels on myocardial infarct size in humans. Results of phase I thrombolysis in myocardial infarction Results of phase I thrombolysis in myocard
(TIMI) trial. Circulation 1991;83:739-46.

5 The ISIS-2 collaborative group. Second International Study of Infarct Survival. Lancet 1988;ii:349-60. 
6 Braunwald E, Pfeffer MA. Ventricular enlargement and remodelling following acute myocardial infarction: mechanisms and $1-6 \mathrm{D}$

7 Stillwell GK. The Law of Laplace. Some clinical applications. Mayo Clin Proc 1973;48:863-9.

8 White HD, Norris RM, Brown MA, et al. Left ventricular end-systolic volume as the major determinant of survival after recovery from myocardial infarction. Circulation 1987, 76:44-51

9 Cleutjens JP, Kandala JC, Guarda E, et al. Regulation of collagen degradation in the rat myocardium after infarction. $\mathcal{F}$ Mol Cell Cardiol 1995;27:1281-92.

10 Hirohata S, Kusachi S, Murakami M, et al. Time dependent alterations of serum matrix metalloproteinase-1 and metalloproteinase-1 tissue inhibitor after successful reperfusion of acute myocardial infarction. Heart 1997;78:27884 .

11 Olivetti G, Capasso JM, Sonnenblick EH, et al. Side-to-side slippage of myocytes participates in ventricular wall slippage of myocytes participates in ventricular wall remodeling acutely

12 McAlpine HM, Morton JJ, Leckie B, et al. Neuroendocrine activation after acute myocardial infarction. Br Heart $\mathcal{F}$

13 Packer $M$. The neurohormonal hypothesis: a theory to explain the mechanism of disease progression in heart failure. $f$ Am Coll Cardiol 1992;20:248-54.

14 Sugden PH. Signalling in myocardial hypertrophy-life after calcineurin? Circ Res 1999;84:633-46.

15 Yusuf S, Peto R, Lewis J, et al. Beta blockade during and after myocardial infarction: an overview of the randomized trials. Prog Cardiovasc Dis 1985;27:335-71.

16 Packer M, Bristow MR, Cohn JN, et al. The effect of carvedilol on morbidity and mortality in patients with chronic heart failure. US Carvedilol Heart Failure Study Group. N Engl f Med 1996;334:1349-55.

17 CIBIS-II Investigators and Committees. The Cardiac Insufficiency Study II (CIBIS-II): a randomised trial. Lancet ficiency Study

18 GISSI-3: Gruppo Italiano per lo Studio della Sopravvivenza nell'Infarto Miocardico. Effects of lisinopril and transdermal glyceryl trinitrate singly and together on 6-week mortality and ventricular function after acute myocardial infarction. Lancet 1994;343:1115-22.

19 Pfeffer MA, Braunwald E, Moye LA, et al. Effect of captopril on mortality and morbidity in patients with left ventricular dysfunction after myocardial infarction. Results of the survival and ventricular enlargement trial. The SAVE Investigators. N Engl 7 Med 1992;327:669-77.

20 The SOLVD Investigators. Effect of enalapril on survival in patients with reduced left ventricular ejection fractions and congestive heart failure. N Engl f Med 1991;325:293-302

21 Goodfriend TL, Elliot ME, Catt KJ. Angiotensin receptors and their antagonists. $N$ Engl f Med 1996;334:1649-54.

22 Pitt B, Segal R, Martinez FA, et al. Randomised trial of losartan versus captopril in patients over 65 with heart failure (evaluation of Losartan in the Elderly Study, ELITE). Lancet 1997;349:747-52.

23 Struthers AD. Ten years of natriuretic peptide research: new dawn for their diagnostic and therapeutic use? $B M \mathcal{F}$ 1994;308:1615-19.
24 Lang CC, Struthers AD. Atrial and brain natriuretic peptides-a dual natriuretic peptide system potentially nvolved in circulatory homeostasis. Clin Sci 1992;83:51927

25 Trippodo NC, Fox M, Monticello TM, et al. Simultaneous inhibition of neutral endopeptidase and angiotensin converting enzyme by omapatrilat increased survival in heart failure hamsters greater than with ACE inhibition. Circulation 1998;98(suppl I):4095.

26 Levin ER. Endothelins. N Engl F Med 1995;333:356-63.

27 Watanabe T, Suzuki N, Shimamoto N, et al. Endothelin in myocardial infarction. Nature 1990;344:114.

28 Mulder P, Richard V, Derumeaux G, et al. Role of endogenous endothelin in chronic heart failure: effect of long-term treatment with an endothelin antagonist on survival, hemodynamics, and cardiac remodeling. Circulation 1997;96:1976-82.

29 Packer M, Caspi A, Charlon V, et al. Multi-centre, doubleblind, placebo-controlled study of long-term endothelin blockade with bosentan in chronic heart failure - results of the REACH-1 trial. Circulation 1998;98(suppl I):12.

30 Colucci WS. Molecular and cellular mechanisms of myocardial failure. Am f Cardiol 1997;80(suppl A):15$25 \mathrm{~L}$.

31 Simpson PC. Role of proto-oncogenes in myocardial hypertrophy. Am f Cardiol 1988;62(suppl G):13-19G.

32 Iwaki K, Sukhatme VP, Shubeita HE, et al $\alpha$-and $\beta$-adrenergic stimulation induces distinct patterns of immediate gene expression in neonatal rat myocardial cells. f Biol Chem 1990;265:13809-17.

33 Adinolfi A, Adinolfi M, Lessof MH. Alpha-feto-protein during development and in disease. F Med Gen 1975;12:13851.

34 Vannan MA, Taylor DJ. Ventricular remodelling after myocardial infarction. Br Heart f 1992;68:257-9.

35 Marber MS, Brown DL, Kloner RA. The open artery hypothesis: to open, or not to open, that is the question. Eur Heart f 1996;17:505-9.

36 Hochman JS, Choo H. Limitation of myocardial infarct expansion by reperfusion independent of myocardial salvage. Circulation 1987;75:299-306.

37 White HD, Cross DB, Elliot JM, et al. Long-term prognostic importance of patency of the infarct-related coronary artery after thrombolytic therapy for acute myocardial infarction. Circulation 1994;89:61-7.

38 The SWIFT investigators (Should We Intervene Following Thrombolysis?). SWIFT trial of delayed elective intervention $v$ conservative treatment after thrombolysis with anistreplase in acute myocardial infarction. BMF 1991;302: 555-60.

39 Williams DO, Braunwald E, Knatterud GK, et al. One-year results of the thrombolysis in myocardial infarction investigation (TIMI) phase II trial. Circulation 1992;85:533-42.

40 Horie $\mathrm{H}$, Takahashi M, Minai K, et al. Long-term beneficial effect of late reperfusion for acute anterior myocardial infarction with percutaneous transluminal coronary angioplasty. Circulation 1998;98:2377-82. 